\title{
Influencia del tipo de suelo en la características de los mostos, vinos y destilados del Cv. L Palomino fino
}

\section{Influence of soil on the characteristics of musts, wines and distillates from cv. L Palomino fino}

\author{
$\mathrm{M}^{\mathrm{a}}$ Victoria Jimenez-Povedano ${ }^{1}$, Emma Cantos-Villar ${ }^{1}, \mathrm{M}^{\mathrm{a}}$ Jesús Jimenez-Hierro $^{1}$, José Alberto Casas ${ }^{2}$, Luis Miguel \\ Trillo $^{2}$, Salvador Guimera ${ }^{2}$ y Belén Puertas ${ }^{1 *}$ \\ ${ }^{1}$ Instituto de Investigación y Formación Agraria y Pesquera (IFAPA), Centro Rancho de la Merced. Consejería de \\ Agricultura, Pesca y Desarrollo Rural. Cañada de la Loba (CA-3102) PK 3.1. 11471 Jerez de la Frontera. Spain. \\ ${ }^{2}$ Centro de Investigación, Desarrollo, Innovación y Medio Ambiente (CIDIMA). Bodegas González Byass Jerez, SLU. C/ \\ Manuel María González no 12. 11470 Jerez de la Frontera. Spain.
}

\begin{abstract}
Resumen. Tradicionalmente los Brandis de Jerez se elaboran con destilados o aguardientes de vinos de la variedad Airén. Sin embargo, algunas bodegas del marco de Jerez están apostando por la elaboración de Brandis a partir de la variedad Palomino fino, mayoritaria en el marco de Jerez. En este trabajo se ha estudiado la composición química y se ha realizado el análisis sensorial de mostos, vinos y destilados procedentes de uva de la variedad Palomino fino cultivada en tres tipos de suelos diferentes: albarizas, barros y arenas. Los mostos de la uva cultivada en albarizas presentaron mayor grado sacarimétrico, más acidez total y menor $\mathrm{pH}$. Consecuentemente con los mostos de partida, fueron los vinos procedentes de la uva cultivada en suelo de albarizas los que presentaron mayor grado alcohólico. Los vinos procedentes de la uva cultivada en suelo de barros presentaron más concentración de acetato de etilo y de n-propanol. Sensorialmente los vinos mejor valorados fueron los procedentes de la uva cultivada en suelo de barros. Respecto a los destilados, los mejor valorados sensorialmente fueron los procedentes de uva cultivada en suelo de albarizas.
\end{abstract}

\begin{abstract}
Brandies are usually elaborated from white grapevine Airen. However, wineries are currently trying to wide the brandies production testing other varieties. Palomino fino variety is mainly cultivated in albarizas soil, and in minor proportion in mud and sandy soils. In the current work, the analytical composition of Palomino fino musts, wines and distillates, which have been cultivated in albarizas, mud and sands soils, has been studied. The must from grape cultivated in albarizas soil highlighted for showing high sugar content and total acidity, meanwhile the $\mathrm{pH}$ the lowest value. The wine come from grape cultivated in albarizas showed the highest ethanol content, in agreement with sugar content in the must. The wines from the grapes grown in mud soil had a higher concentration of ethyl acetate and n-propanol. In tasting, the wines come from grape cultivated in mud soil obtaining the highest score in fruit aroma intensity. Regarding distillates, those come from albarizas soil showed the highest scores.
\end{abstract}

\section{Introducción}

La calidad de los aguardientes de vino de baja graduación, denominados tradicionalmente holandas, viene determinada, principalmente, por las materias primas de partida y por cómo se realice el proceso de elaboración. Así, la calidad del Brandy de Jerez viene determinada por los destilados de vino utilizados para su obtención.

Al producirse alguna modificación en el proceso de elaboración o en la misma materia prima se podrían obtener nuevos productos con peculiaridades $\mathrm{y}$ características diferentes a los ya existentes diversificando así la producción [1].
Estas modificaciones pueden ser, entre otras, la variedad de uva, la temperatura de fermentación de los vinos, la fecha de vendimia, la zona de cultivo o la presencia de lías en el proceso de destilación. Todos, o algunos de ellos, podrían conducir a la obtención de productos de calidad diferenciada o bien nuevos productos para su posterior comercialización tras su envejecimiento en madera.

En este trabajo, se muestran los resultados de los análisis químico-enológicos y sensoriales de los vinos y destilados de la variedad Palomino fino, de la cosecha de 2017, procedentes de uva cultivada en distintos tipos de suelos, como son: albarizas, barros y arenas.

En la cosecha de 2016 se realizó un estudio 
preliminar, a escala de laboratorio, en la bodega González Byass.

\section{Material y Métodos}

\subsection{Características del ensayo y material vegetal}

Los tres tipos de suelos de donde procede la uva para este estudio son: albarizas, barros y arenas.

El suelo de albarizas se caracteriza por su elevado contenido en carbonato cálcico, con entre un 25 y un 40 $\%$ de caliza activa, pobre en materia orgánica y en nitrógeno y alto nivel de porosidad, lo que ayuda a retener la humedad. Los suelos de barros se ubican en valles y vaguadas, se componen de arcilla, arena, caliza y materia orgánica descompuesta, tienen un color más oscuro y mayor fertilidad. Los de arenas se localizan en las zonas costeras, se componen de arena, arcilla y menos del $20 \%$ de caliza activa [2].

En todos los casos la variedad cultivada es la Palomino fino, que ocupa más del $95 \%$ de la superficie de vid cultivada en el marco de Jerez. Esta variedad es idónea para la elaboración de los vinos generosos de la zona. Actualmente, se está estudiando su aptitud para obtener holandas de calidad aptas para envejecer como brandis de Jerez.

En la Tabla 1 se muestra, para cada tipo de suelo el municipio, el pago, la finca, la parcela, el tipo de poda y la edad del viñedo.

Tabla 1. Producción de uva y parámetros de los mostos.

\begin{tabular}{|l|c|c|c|}
\hline & Albarizas & Barros & Arenas \\
\hline Municipio & $\begin{array}{c}\text { Jerez de la } \\
\text { Frontera }\end{array}$ & $\begin{array}{c}\text { Jerez de la } \\
\text { Frontera }\end{array}$ & $\begin{array}{c}\text { Sanlúcar de } \\
\text { Barrameda }\end{array}$ \\
\hline Pago & Carrascal & Carrascal & La Loma alta \\
\hline Finca & La Canariera & La Canariera & Las Lomas \\
\hline Parcela & Esteve & Canariera & Las Lomas \\
\hline Poda & Vara y pulgar & Cordón doble & Vara y Pulgar \\
\hline $\begin{array}{l}\text { Edad de la } \\
\text { viña (años) }\end{array}$ & 31 & 26 & 30 \\
\hline
\end{tabular}

\subsection{Controles enológicos}

\subsubsection{Vinificación}

La vendimia se realizó manualmente a finales de agosto, en fechas similares para los tres tipos de suelos, con el fin de poder comparar los resultados.

La uva se trasladó a la bodega experimental del Rancho de la Merced, en cajas de plástico perforadas, de unos $18 \mathrm{Kg}$ de capacidad. Se despalilló y se prensó en prensa neumática. Al mosto, a la salida de la prensa, se le adicionaron 2,5 $\mathrm{ml} / \mathrm{Hl}$ de enzimas pectolíticas (Blanco L, Sepsa-Enartis) y se introdujo en una cámara frigorífica a $4^{\circ} \mathrm{C}$ para su desfangado, donde se mantuvo 24 horas. No se adicionó anhídrido sulfuroso ya que el destino de estos vinos era la destilación. En los mostos desfangados se determinaron los siguientes parámetros: grado Brix, acidez total, pH, ácido tartárico, ácido málico e índice de Folin-Ciocalteu (IFC).

Tras el desfangado, el mosto se encubó en 2 depósitos de $50 \mathrm{~L}$ de capacidad, de acero inoxidable, provistos de sistema de refrigeración por ducha. Se corrigió la acidez con ácido tartárico hasta llevar el $\mathrm{pH}$ a 3,3 y se adicionaron $25 \mathrm{~g} / \mathrm{Hl}$ de levaduras comerciales previamente hidratadas (Viniferm BY, Agrovin S.A.).

La temperatura de fermentación se estableció en 18 ${ }^{\circ} \mathrm{C}$. Diariamente se midió el grado Baumé, la densidad y la temperatura de los mostos. Al alcanzar densidad inferior a $1000 \mathrm{~g} / \mathrm{ml}$ se desliaron y se mantuvieron en cámara frigorífica a $4^{\circ} \mathrm{C}$ hasta su destilación.

Tras el deslío se determinaron a los vinos los parámetros químico-enológicos de calidad. Los análisis de los mostos y vinos han sido realizados siguiendo los métodos internacionales de análisis de la OIV [3].

\subsubsection{Destilación}

Los vinos se destilaron de forma discontinua simple (un único proceso de destilación para cada vino) en alquitara de cobre. De cada vino se realizaron dos destilaciones.

El calentamiento se llevó a cabo por fuego directo utilizando como combustible gas propano y la refrigeración se realizó por circulación de agua fría. De esta manera, la temperatura de salida del destilado fue inferior a $15^{\circ} \mathrm{C}$. Se destilaron por duplicado en lotes de 12 L. Se recogieron distintas fracciones a lo largo del proceso de destilación. En base al análisis por cromatografía de gases (GC), se definieron las fracciones de cabeza y corazón también llamadas "holanda". Por otra parte, la fracción de cola se definió en base al grado alcohólico. En todos los casos, el porcentaje de la fracción de cabezas respecto al volumen de vino inicial de partida fue de un $1 \%$. La destilación se consideró terminada cuando el grado alcohólico del destilado obtenido fue inferior a $20 \%$ vol.

Las diferentes fracciones obtenidas de los destilados fueron almacenadas en frio hasta su posterior análisis en el laboratorio. Los análisis de los destilados han sido realizados siguiendo los métodos internacionales de análisis de la OIV [4].

Las determinaciones analíticas realizadas a las fracciones de cabezas, corazones y colas de los destilados fueron: grado alcohólico, $\mathrm{pH}$, sulfuroso total y cobre. Asimismo, se identificaron y cuantificaron por CG a los corazones u holandas los siguientes compuestos volátiles: acetaldehído, acetona, acetato de metilo, acetato de etilo, 1-1-dietoxietano, metanol, 2-butanol, butirato de etilo, npropanol, isobutanol, alilico, acetato de isoamilo, nbutanol, isoamílicos, 2-hexanol, hexanoato de etilo, heptanoato de etilo, lactato de etilo, n-hexanol, cis-3hexen-1-ol, octanoato de etilo, ácido acético, nonanoato de etilo, linalol, decanoato de etilo, succinato de dietilo, $\alpha$-terpineol, undecanoato de etilo, acetato 2-fenil-etilo, laurato de etilo, 2-fenil-etanol y miristato de etilo.

\subsubsection{Análisis organoléptico de los vinos}

Primeramente se realizaron catas triangulares, en copas negras, para ver si se detectaban, olfativamente, diferencias entre las réplicas de cada ensayo. Para los vinos procedentes de cada tipo de suelo se pusieron 6 puestos y se contó con 12 jueces. En cada puesto había dos copas iguales y una distinta.

El análisis sensorial descriptivo de los vinos se realizó mediante un panel de 7 catadores entrenados en nuestro Centro y 5 catadores de la Bodega González Byass, siendo los jueces los que reflejaron en las 
correspondientes fichas de cata la percepción de los parámetros encontrados en los vinos en cuanto a la sensación visual, olfativa y gustativa. La valoración de cada atributo se realizó en una escala del 0 al 10 , considerando las puntuaciones cercanas al 0 ausentes y las cercanas al 10 muy presentes.

\subsubsection{Análisis organoléptico de los destilados}

El análisis sensorial de los destilados se realizó con el mismo panel de catadores que para los vinos. Los jueces reflejaron en las correspondientes fichas de cata la percepción de los parámetros encontrados en los destilados en cuanto a limpidez en la fase visual y a intensidad, punzante, fragancia, afrutado, vinoso, floral y calidad, en la fase olfativa. La valoración de cada atributo se realizó en una escala del 0 al 8 , considerando las puntuaciones cercanas al 0 ausentes y las cercanas al 8 muy presentes.

\subsection{Análisis estadístico}

Se realizo un análisis de la varianza (ANOVA) para cada determinación analítica, utilizando el programa Statistix software (v. 9.0 Analitycal software, FL, USA).

\section{Resultados y Discusión}

\subsection{Mostos}

En la Tabla 2 se muestra, para cada tipo de suelo, la producción de uva y los parámetros analizados a los mostos desfangados.

Tabla 2. Producción de uva y parámetros de los mostos desfangados.

\begin{tabular}{|l|c|c|c|}
\hline & Albarizas & Barros & Arenas \\
\hline Producción $(\mathrm{Kg} / \mathrm{ha})$ & 8.000 & 10.000 & 10.000 \\
\hline Grado Brix & 22,5 & 20,2 & 18,7 \\
\hline $\mathrm{pH}$ & 3,72 & 3,87 & 3,83 \\
\hline Acidez total $\left(\mathrm{g} / \mathrm{L} \mathrm{TH}_{2}\right)$ & 2,94 & 2,60 & 2,72 \\
\hline Ácido tartárico $(\mathrm{g} / \mathrm{L})$ & 4,92 & 5,32 & 5,12 \\
\hline Ácido málico $(\mathrm{g} / \mathrm{L})$ & 0,41 & 0,45 & 0,62 \\
\hline Índice Folin-Ciocalteu & 7,24 & 4,55 & 5,90 \\
\hline
\end{tabular}

La menor producción de uva y la mayor graduación Brix se obtuvo cuando se cultivó en suelo de albarizas. Asimismo, los mostos procedentes de uva cultivada en albarizas fueron los que presentaron menor valor de $\mathrm{pH}$ y mayor acidez total. El menor grado Brix fue el de la uva cultivada en suelo de arenas, con la misma producción de uva que la cultivada en suelo de barros.

La variedad Palomino fino se caracteriza por valores bajos de ácido málico [5], se observó que cuando se cultiva en suelo de arenas la cantidad este ácido es superior que cuando se cultiva en suelo de albarizas o de barros.

Respecto al índice de Folin-Ciocalteu los valores más altos se obtuvieron cuando la uva se cultivó en suelo de albarizas y los más bajos cuando la uva procedía de suelo de barros.

\subsection{Vinos}

En la Figura 1 se muestra la cinética de la fermentación alcohólica (FA). La FA de los mostos procedentes de suelo de albarizas duró 15 días, mientras que los procedentes de suelo de barros y de arenas duraron 8 y 10 días respectivamente. Hay que tener en cuenta que el grado Brix de los mostos de suelo de albarizas era más elevado. Todas las fermentaciones se desarrollaron correctamente y los vinos quedaron con contenido en azucares reductores inferiores a $2 \mathrm{~g} / \mathrm{L}$.

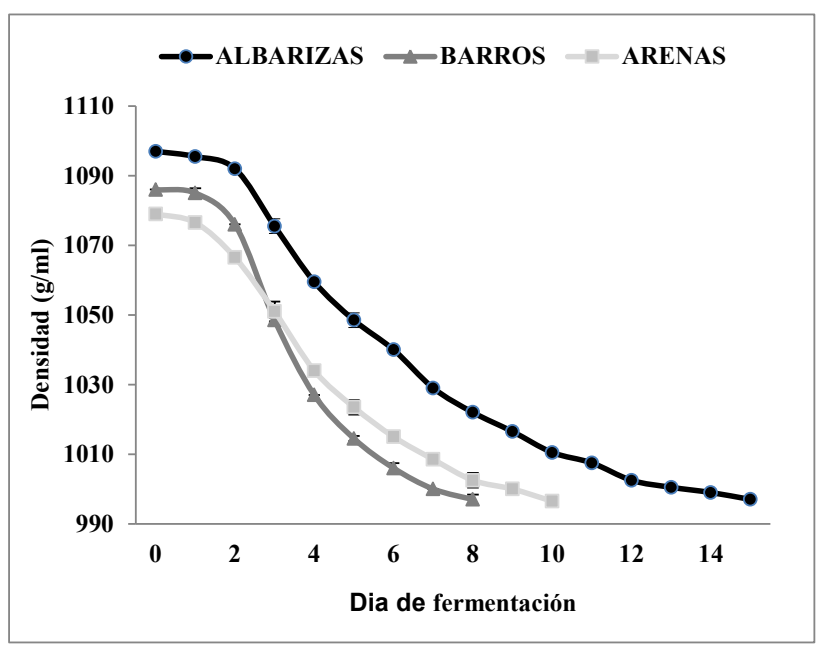

Figura 1. Evolución de la fermentación.

Los resultados de las determinaciones analíticas de los vinos se muestran en la Tabla 3.

Los grados alcohólicos de los vinos están en concordancia con los grados Brix de los mostos de partida, siendo significativamente más altos los de los vinos que procedían de uva cultivada en suelo de albarizas. En concordancia está la densidad relativa, es decir, valores más bajos a mayor concentración de etanol. Generalmente, los vinos de mayor graduación alcohólica presentan valores más altos de glicerol, por lo que los vinos procedentes de uva cultivada en suelo de albarizas presentaron valores más altos de dicho compuesto.

No se encontraron diferencias significativas ni en azúcares ni en el extracto seco. Todos ellos presentaron valores bajos de acidez volátil, lo que indicaba ausencia e desviaciones en la fermentación alcohólica.

Respecto a los ácidos orgánicos, no hubo diferencias significativas en los valores del ácido acético ni del ácido cítrico. Los vinos que presentaron valores más bajos de ácido succínico fueron los procedentes de uva cultivada en suelo de barros. Los vinos procedentes de uva cultivada en suelo de arenas realizaron la fermentación maloláctica, por lo que presentaron los valores más altos de ácido láctico y más bajos de ácido málico.

En cuanto a compuestos volátiles mayoritarios no se encontraron diferencias significativas ni en acetaldehído, ni en isoamílicos, ni en el sumatorio de alcoholes superiores. Los vinos procedentes de uva cultivada en suelo de barros fueron los que presentaron valores más altos de acetato de etilo, metanol y n-propanol y los procedentes de uva cultivada en suelo de albarizas los que presentaron mayor concentración de isobutanol.

Los vinos con tonalidades más verdosas, es decir valores más bajos de $\mathrm{a}^{*}$ fueron los procedentes de uva cultivada en suelo de barros y los de mayor tonalidad amarilla fueron los procedentes de uva cultivada en 
albarizas, mayor valor de b* y de absorbancia a $420 \mathrm{~nm}$.

Las concentraciones de los metales están dentro de las permitidas en vinos blancos jóvenes. Cabe destacar que los vinos procedentes de uva cultivada en suelo de arenas presentaron valores más altos de sodio y de hierro.

Tabla 3. Determinaciones analíticas de los vinos.

\begin{tabular}{|c|c|c|c|c|}
\hline & Albarizas & Barros & Arenas & G.S. \\
\hline \multicolumn{5}{|c|}{ Parámetros convencionales } \\
\hline Etanol $(\% \mathrm{v} / \mathrm{v})$ & $14,69 \mathrm{a}$ & $12,51 \mathrm{~b}$ & $11,48 \mathrm{c}$ & *** \\
\hline Densidad relativa & $0,9871 \mathrm{c}$ & $0,9895 \mathrm{~b}$ & $0,9906 \mathrm{a}$ & *** \\
\hline Azúcares $(\mathrm{g} / \mathrm{L})$ & 1,97 & 1,36 & 1,12 & n.s. \\
\hline Ac. total $\left(\mathrm{g} / \mathrm{L} \mathrm{TH}_{2}\right)$ & $3,91 \mathrm{a}$ & $3,27 \mathrm{~b}$ & $3,75 \mathrm{a}$ & $*$ \\
\hline Ac. volátil $(\mathrm{g} / \mathrm{L}$ AcH $)$ & $0,22 \mathrm{a}$ & $0,12 \mathrm{~b}$ & $0,13 \mathrm{~b}$ & $* * *$ \\
\hline $\mathrm{pH}$ & $3,25 \mathrm{~b}$ & $3,36 \mathrm{a}$ & $3,29 \mathrm{~b}$ & ** \\
\hline Extracto seco $(\mathrm{g} / \mathrm{L})$ & 12,55 & 13,05 & 12,23 & n.s. \\
\hline Glicerol $(\mathrm{g} / \mathrm{L})$ & $7,10 \mathrm{a}$ & $5,98 \mathrm{~b}$ & $6,06 \mathrm{~b}$ & $*$ \\
\hline \multicolumn{5}{|c|}{ Ácidos orgánicos (g/L) } \\
\hline Acético & 0,19 & 0,04 & 0,10 & n.s. \\
\hline Cítrico & 0,05 & 0,09 & 0,03 & n.s. \\
\hline Succínico & $0,73 \mathrm{a}$ & $0,29 \mathrm{~b}$ & $0,75 \mathrm{a}$ & * \\
\hline Tartárico & $1,96 \mathrm{~b}$ & $2,17 \mathrm{a}$ & $2,10 \mathrm{a}$ & * \\
\hline Málico & $0,41 \mathrm{a}$ & $0,31 \mathrm{a}$ & $0,06 \mathrm{~b}$ & $* *$ \\
\hline Láctico & $0,03 \mathrm{c}$ & $0,12 \mathrm{~b}$ & $0,54 \mathrm{a}$ & *** \\
\hline \multicolumn{5}{|c|}{ Compuestos volátiles mayoritarios (ppm) } \\
\hline Acetaldehído & 24,5 & 22,9 & 25,2 & n.s. \\
\hline Acetato de etilo & $41,0 \mathrm{~b}$ & $71,0 \mathrm{a}$ & $35,5 \mathrm{~b}$ & $*$ \\
\hline Metanol & $28,4 \mathrm{~b}$ & $35,4 \mathrm{a}$ & $30,9 \mathrm{~b}$ & * \\
\hline N-propanol & $14,7 \mathrm{c}$ & $26,2 \mathrm{a}$ & $16,4 \mathrm{~b}$ & *** \\
\hline Isobutanol & $13,8 \mathrm{a}$ & $11,2 \mathrm{~b}$ & $10,6 \mathrm{~b}$ & * \\
\hline Isoamílicos & 169,5 & 131,8 & 137,8 & n.s. \\
\hline Alcoholes superiores & 198,0 & 169,1 & 164,7 & n.s. \\
\hline \multicolumn{5}{|c|}{ Parámetros relacionados con el color } \\
\hline $\mathrm{L}$ & 96,49 & 97,98 & 97,26 & n.s. \\
\hline $\mathrm{a}^{*}$ & $-1,34 \mathrm{a}$ & $-1,65 b$ & $-1,40 \mathrm{a}$ & $*$ \\
\hline $\mathrm{b}^{*}$ & $13,64 \mathrm{a}$ & $9,96 \mathrm{~b}$ & $10,78 \mathrm{~b}$ & * \\
\hline $\mathrm{C}_{\mathrm{ab}}$ & $13,71 \mathrm{a}$ & $9,99 \mathrm{~b}$ & $10,87 \mathrm{~b}$ & * \\
\hline $\mathrm{h}$ & $95,61 \mathrm{c}$ & 99,49 a & $97,36 \mathrm{~b}$ & ** \\
\hline D.O. $420 \mathrm{~nm}$ & $0,096 \mathrm{a}$ & $0,064 \mathrm{~b}$ & $0,075 \mathrm{~b}$ & ** \\
\hline D.O. $520 \mathrm{~nm}$ & 0,020 & 0,011 & 0,015 & n.s. \\
\hline \multicolumn{5}{|c|}{ Metales (ppm) } \\
\hline Calcio & 26 & 24 & 30 & n.s. \\
\hline Sodio & $14,5 \mathrm{~b}$ & $11,0 \mathrm{c}$ & $17,5 \mathrm{a}$ & ** \\
\hline Potasio & $419 \mathrm{~b}$ & $511 \mathrm{a}$ & $522 \mathrm{a}$ & $* * *$ \\
\hline Hierro & $0,27 \mathrm{~b}$ & $0,28 \mathrm{~b}$ & $0,59 \mathrm{a}$ & ** \\
\hline Cobre & 0,52 & 0,58 & 0,44 & n.s. \\
\hline Zinc & 0,18 & 0,18 & 0,20 & n.s. \\
\hline
\end{tabular}

Dentro de cada fila, letras diferentes indican diferencias significativas a $\mathrm{P} \leq 0,05$ según el test LSD. G.S.: grado de significación; n.s.: no significativo; *: significativo a $\mathrm{P} \leq 0,05 ; * *$ : significativo a $\mathrm{P} \leq 0,01$; ***: significativo a $\mathrm{P} \leq 0,001$.

\subsection{Destilados}

En las Tablas 4, 5 y 6 se muestran los parámetros físico-químicos de las tres fracciones de los destilados: cabezas, corazones y colas. Como se ha comentado anteriormente los cortes de cabeza se realizaron en el mismo punto para todos los destilados (1\% del vino inicial), es decir $120 \mathrm{ml}$. El \% de etanol más alto, en las fracciones de cabeza, fue el de los destilados procedentes de uva cultivada en suelo de albariza, al igual que los correspondientes vinos de partida.

En todas las fracciones (cabezas, corazones y colas) de cada destilado las determinaciones de $\mathrm{pH}$, cobre y $\mathrm{SO}_{2}$ total estuvieron dentro de los rangos esperados. En todos los casos, el cobre tuvo el mismo comportamiento, aumentó de cabeza a corazón y de corazón a cola siendo el último aumento más pronunciado que el primero.

Los caudales de destilación, como es lógico, disminuyeron de las fracciones de cabezas a las de corazones y de las de corazones a las de colas, manteniendo la temperatura de salida del destilado prácticamente constante a lo largo de cada destilación.

Tabla 4. Parámetros físico-químicos de las cabezas.

\begin{tabular}{|l|c|c|c|}
\hline & Albarizas & Barros & Arenas \\
\hline Etanol $(\% \mathrm{v} / \mathrm{v})$ & 77,45 & 75,47 & 76,50 \\
\hline $\mathrm{pH}$ & 5,95 & 5,93 & 5,27 \\
\hline Cobre $(\mathrm{ppm})$ & 2,63 & 2,11 & 5,60 \\
\hline $\mathrm{SO}_{2}$ total $(\mathrm{ppm})$ & 4 & 4 & 7 \\
\hline Volumen $(\mathrm{ml})$ & 120 & 120 & 120 \\
\hline Caudal $(\mathrm{ml} / \mathrm{min})$ & 32 & 30 & 29 \\
\hline $\mathrm{T}^{\mathrm{a}}$ salida destilado $\left({ }^{\circ} \mathrm{C}\right)$ & 11 & 11 & 11 \\
\hline
\end{tabular}

Tabla 5. Parámetros físico-químicos de los corazones.

\begin{tabular}{|l|c|c|c|}
\hline & Albarizas & Barros & Arenas \\
\hline Etanol (\% v/v) & 66,50 & 65,57 & 68,00 \\
\hline $\mathrm{pH}$ & 5,95 & 5,92 & 5,13 \\
\hline Cobre $(\mathrm{ppm})$ & 2,98 & 2,52 & 8,13 \\
\hline $\mathrm{SO}_{2}$ total $(\mathrm{ppm})$ & 3 & 4 & 8 \\
\hline Volumen $(\mathrm{ml})$ & 2000 & 1250 & 1250 \\
\hline Caudal $(\mathrm{ml} / \mathrm{min})$ & 27 & 25 & 26 \\
\hline $\mathrm{T}^{\mathrm{a}}$ salida destilado $\left({ }^{\circ} \mathrm{C}\right)$ & 10 & 10 & 10 \\
\hline
\end{tabular}

Tabla 6. Parámetros físico-químicos de las colas.

\begin{tabular}{|l|c|c|c|}
\hline & Albarizas & Barros & Arenas \\
\hline Etanol (\% v/v) & 39,1 & 40,5 & 41,5 \\
\hline $\mathrm{pH}$ & 5,27 & 5,41 & 5,33 \\
\hline Cobre $(\mathrm{ppm})$ & 9,93 & 7,53 & 9,16 \\
\hline $\mathrm{SO}_{2}$ total $(\mathrm{ppm})$ & 4 & 5 & 4 \\
\hline Volumen $(\mathrm{ml})$ & 450 & 650 & 700 \\
\hline Caudal $(\mathrm{ml} / \mathrm{min})$ & 19 & 19 & 20 \\
\hline $\mathrm{T}^{\mathrm{a}}$ salida destilado $\left({ }^{\circ} \mathrm{C}\right)$ & 10 & 10 & 10 \\
\hline
\end{tabular}

Para una "holanda" de calidad el grado alcohólico de los corazones debe estar comprendido entre 65 y $70 \%$ v/v. Al unir las diferentes fracciones de los destilados para obtener el grado alcohólico deseado en los corazones $\mathrm{u}$ holandas $(6570 \% \mathrm{v} / \mathrm{v})$, los volúmenes finales fueron mucho mayores en los destilados de vinos procedentes de uva cultivada en suelo de albariza, respecto a los destilados de vinos procedentes de uva cultivada en suelos de barros o de arenas $(2000 \mathrm{ml}$ frente a $1250 \mathrm{ml})$, debido a la mayor graduación alcohólica de estos vinos.

El grado alcohólico de las fracciones de colas estuvo en torno al $40 \% \mathrm{v} / \mathrm{v}$, alcanzando un volumen de éstas menor en los destilados procedentes de la uva cultivada en suelo de albarizas.

En la Tabla 7 se muestran las concentraciones de aldehídos, cetonas y alcoholes de las fracciones corazón de los destilados. No se encontraron diferencias significativas en acetaldehído, cuyo descriptor es oxidación, estando todos correctos con valores inferiores a $70 \mathrm{~g} / \mathrm{Hl}$ a.a.

Cuando la relación n-propanol/isobutanol de los aguardientes es $\geq 1$ es síntoma de alguna alteración bacteriana en los vinos de partida. En este trabajo los destilados que presentan una relación mayor entre estos 
compuestos son los procedentes de suelo de arenas.

Los destilados de uva cultivada en suelo de arenas presentaron valores más altos de metanol, 2-butanol, npropanol, n-butanol, 2-hexanol y cis-3-hexen-1-ol.

Tabla 7. Aldehídos, cetonas y alcoholes de los corazones

(g/Hl a.a.).

\begin{tabular}{|l|c|c|c|c|}
\hline & Albarizas & Barros & Arenas & G.S. \\
\hline Acetaldehído & 17,73 & 34,40 & 17,43 & n.s. \\
\hline Acetona & $0,385 \mathrm{a}$ & $0,281 \mathrm{c}$ & $0,344 \mathrm{~b}$ & $* * *$ \\
\hline Metanol & $15,33 \mathrm{c}$ & $19,45 \mathrm{~b}$ & $20,68 \mathrm{a}$ & $* * *$ \\
\hline 2-Butanol & $0,235 \mathrm{~b}$ & $0,253 \mathrm{~b}$ & $0,318 \mathrm{a}$ & $* *$ \\
\hline n-Propanol & $10,88 \mathrm{c}$ & $16,66 \mathrm{~b}$ & $23,80 \mathrm{a}$ & $* * *$ \\
\hline Isobutanol & 10,25 & 11,10 & 10,50 & n.s. \\
\hline n-Butanol & $0,459 \mathrm{~b}$ & $0,375 \mathrm{c}$ & $0,575 \mathrm{a}$ & $* * *$ \\
\hline Isoamílicos & $134 \mathrm{~b}$ & $157 \mathrm{a}$ & $135 \mathrm{~b}$ & $* *$ \\
\hline 2-Hexanol & $0,369 \mathrm{~b}$ & $0,378 \mathrm{~b}$ & $0,500 \mathrm{a}$ & $* *$ \\
\hline n-Hexanol & $0,467 \mathrm{~b}$ & $1,080 \mathrm{a}$ & $0,478 \mathrm{~b}$ & $* * *$ \\
\hline Cis-3-hexen-1-ol & $0,126 \mathrm{~b}$ & $0,114 \mathrm{~b}$ & $0,151 \mathrm{a}$ & $* *$ \\
\hline 2-Feniletanol & $3,21 \mathrm{a}$ & $2,66 \mathrm{a}$ & $1,20 \mathrm{~b}$ & $* * *$ \\
\hline
\end{tabular}

Dentro de cada fila, letras diferentes indican diferencias significativas a $P \leq 0,05$ según el test LSD. G.S.: grado de significación; n.s.: no

significativo; **: significativo a $\mathrm{P} \leq 0,01 ; * * *$ : significativo a $\mathrm{P} \leq$ 0,001

El metanol puede provenir de un prensado excesivo, se considera correcto cuando se encuentra en concentración inferior a $50 \mathrm{~g} / \mathrm{Hl}$ a.a. y en este trabajo están siempre por debajo de dicho valor.

El 2-butanol, cuyo descriptor es fermentación butírica, en el destilado procedente de uva cultivada en suelo de arenas está por encima del valor correcto $(\geq 0,3$ $\mathrm{g} / \mathrm{Hl}$ a.a.) aunque por debajo del inaceptable $(\geq 0,6 \mathrm{~g} / \mathrm{Hl}$ a.a.). El descriptor del n-butanol también es la fermentación butírica, considerándose correcto cuando se encuentra en concentraciones inferiores a $1.0 \mathrm{~g} / \mathrm{Hl}$ a.a., encontrándose este compuesto, en todos los destilados, por debajo de este valor.

El 2-hexanol y el cis-3-hexen-1-ol tienen como descriptores los herbáceos, considerándose inaceptables cuando superan $0,6 \mathrm{~g} / \mathrm{Hl}$ a.a.

Los destilados procedentes de uva cultivada en suelo de barros fueron los que presentaron valores superiores de n-hexanol, cuyo descriptor es resina o hierba verde cortada.

En cuanto al 2-feniletanol, cuyo descriptor es rosa [5], fueron los destilados procedentes de uva cultivada en suelos de albarizas y barros los que presentaron valores más altos.

En las Tablas 8 y 9 se muestran los ésteres (acetatos) y ésteres etílicos superiores.

No se encontraron diferencias significativas en el acetato de metilo cuyo descriptor es disolvente o quitaesmalte, ni en acetato de 2-feniletilo, cuyo descriptor es floral y sotobosque.

El acetato de etilo se encontró en mayor concentración en los destilados procedentes de uva cultivada en suelo de arenas, tiene como descriptor el picado acético y se considera correcto cuando su concentración está por debajo de $455 \mathrm{mg} / \mathrm{L}$ [6].

El descriptor del acetato de isoamilo es fruta madura, plátano y pera, su umbral de percepción es de 1,6 mg/L
[7]. Al igual que el acetato de etilo, se encontró en mayor cantidad en los destilados procedentes de uva cultivada en suelo de arenas.

Tabla 8. Ésteres (acetatos) de las fracciones de corazón (mg/L).

\begin{tabular}{|l|c|c|c|c|}
\hline & Albarizas & Barros & Arenas & G.S. \\
\hline Acetato de metilo & 1,95 & 2,01 & 1,95 & n.s. \\
\hline Acetato de etilo & $159,8 \mathrm{~b}$ & $185,5 \mathrm{~b}$ & $431,5 \mathrm{a}$ & $* * *$ \\
\hline Acetato de isoamilo & $7,57 \mathrm{~b}$ & $6,82 \mathrm{~b}$ & $26,62 \mathrm{a}$ & $* * *$ \\
\hline Acetato 2-feniletilo & 1,53 & 1,24 & 1,75 & n.s. \\
\hline Total acetatos & $\mathbf{1 7 0 , 9} \mathbf{b}$ & $\mathbf{1 9 5 , 7} \mathbf{b}$ & $\mathbf{4 6 1 , 8} \mathbf{a}$ & $* * *$ \\
\hline
\end{tabular}

Dentro de cada fila, letras diferentes indican diferencias significativas a $P \leq 0,05$ según el test LSD. G.S.: grado de significación; n.s.: no significativo; $* * *$ : significativo a $\mathrm{P} \leq 0,001$.

Respecto a los ésteres etílicos no hubo diferencias significativas ni en el hexanoato (caproato), ni en el nonanoato, ni en el decanoato (caprato).

Los que presentaron valores más altos de octanoato (caprilato) fueron los procedentes de uva cultivada en suelo de arenas, cuyos descriptores son fruta madura, manzana, piña, plátano y pera [1].

Tabla 9. Ésteres etílicos superiores de las fracciones de corazón $(\mathrm{mg} / \mathrm{L})$.

\begin{tabular}{|l|c|c|c|c|}
\hline & Albarizas & Barros & Arenas & G.S. \\
\hline Hexanoato de etilo & 0,06 & 0,10 & 0,11 & n.s. \\
\hline Heptanoato de etilo & $0,56 \mathrm{a}$ & $0,51 \mathrm{a}$ & $0,27 \mathrm{~b}$ & $* * *$ \\
\hline Octanoato de etilo & $2,44 \mathrm{~b}$ & $2,79 \mathrm{~b}$ & $4,05 \mathrm{a}$ & $* *$ \\
\hline Nonanoato de etilo & 1,97 & 1,30 & 1,77 & n.s. \\
\hline Decanoato de etilo & 2,16 & 2,35 & 2,57 & n.s. \\
\hline $\begin{array}{l}\text { Total esteres } \\
\text { etílicos superiores }\end{array}$ & $\mathbf{7 , 1 9} \mathbf{b}$ & $\mathbf{7 , 0 7} \mathbf{~ b}$ & $\mathbf{8 , 7 7} \mathbf{a}$ & $* *$ \\
\hline
\end{tabular}

Dentro de cada fila, letras diferentes indican diferencias significativas a $\mathrm{P} \leq 0,05$ según el test LSD. G.S.: grado de significación; n.s.: no significativo; **: significativo a $\mathrm{P} \leq 0,01 ; * * *$ : significativo a $\mathrm{P} \leq$ 0,001 .

\subsection{Análisis organoléptico de los vinos}

En las catas triangulares se detectaron diferencias significativas $(p \leq 0.05)$ entre las repeticiones de los vinos procedentes de la uva cultivada en suelo de albarizas y de la cultivada en suelo de arenas y no hubo diferencias significativas entre los vinos procedentes de uva cultivada en suelo de barros. Al encontrar diferencias significativas entre las repeticiones de los vinos procedentes de uva cultivada en el mismo tipo de suelo se realizaron las catas descriptivas de cada uno de los vinos.

En la Figura 2 se representan los resultados del análisis sensorial de los vinos.

En la fase visual los vinos procedentes de uva cultivada en suelo de arenas estaban algo más turbios que los procedentes de uva cultivada en suelo de albarizas y en suelo de barros. Los puntuados con valores más altos en color fueron los procedentes de uva cultivada en suelo de albarizas, lo que coincide con los datos analíticos mostrados en la Tabla 3 .

En la fase olfativa los vinos mejor valorados fueron los procedentes de uva cultivada en suelo de barros, que fueron puntuados con valores más altos en las notas cítricas, fruta de árbol (manzana y pera), fruta de hueso (albaricoque y melocotón) y fruta madura. 


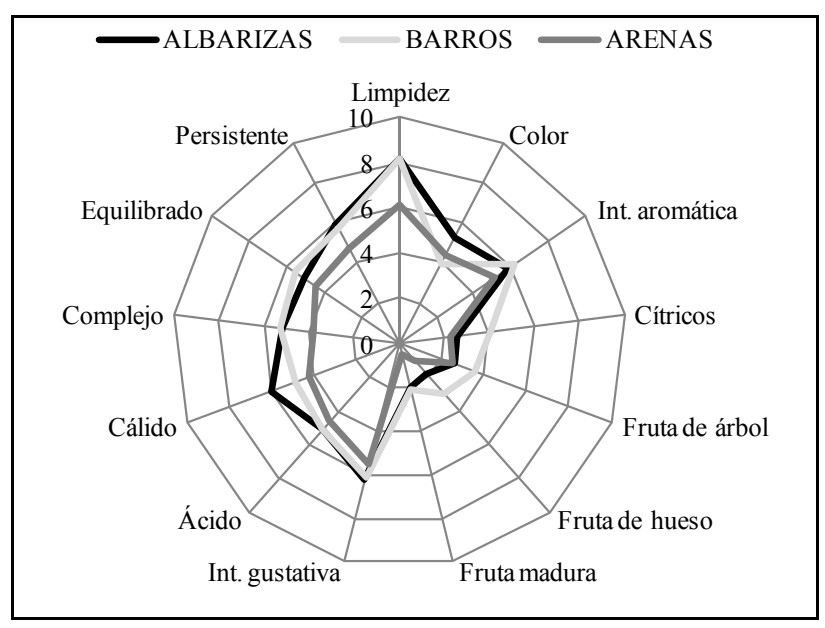

Figura 2. Análisis sensorial descriptivo de los vinos.

En la fase gustativa destacaron los vinos procedentes de uva cultivada en suelo de albarizas por ser más cálidos (mayor grado alcohólico). En cuanto a complejidad, equilibrio y persistencia, los vinos procedentes de uva cultivada en suelo de albarizas y barros alcanzaron puntuaciones similares y más altas que los vinos procedentes de uva cultivada en suelo de arenas.

\subsection{Análisis organoléptico de los destilados}

En la Figura 3 se representan los resultados medios, por tipo de suelo, del análisis sensorial de los destilados. La cata descriptiva se realizó, en las fracciones corazón, de los destilados procedentes de uva cultivada en cada tipo de suelo. Para la cata, los destilados se hidrataron con agua osmotizada, para que tuvieran un $30 \% \mathrm{v} / \mathrm{v}$ de etanol.

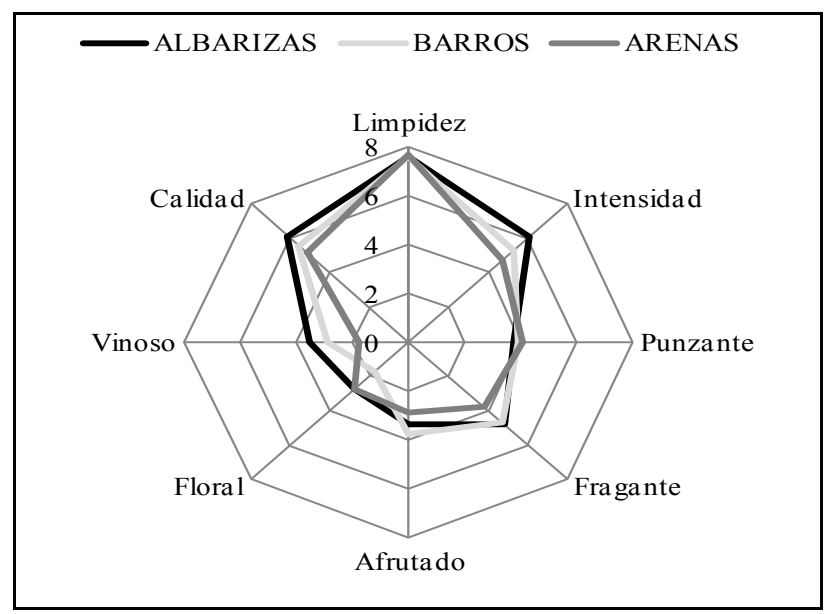

Figura 3. Análisis sensorial descriptivo de los destilados.

Todos ellos alcanzaron idénticas puntuaciones en limpidez.

Los destilados procedentes de uva cultivada en suelo de albarizas fueron los mejor valorados en los atributos: intensidad, vinoso y calidad. Los procedentes de uva cultivada en suelo de barros fueron mejor puntuados en el atributo afrutado.

En general, los que alcanzaron puntuaciones más bajas, fueron los procedentes de uva cultivada en suelo de arenas. Posiblemente las notas afrutadas del acetato de isoamilo, muy presente en los destilados procedentes de uva cultivada en suelo de arenas, se han visto enmascaradas por el acetato de etilo, que se encontraba en concentraciones elevadas en dichos destilados.

\section{Conclusiones}

Los vinos procedentes de uva cultivada en suelos de albarizas fueron los de más color y mayor grado alcohólico y los procedentes de suelos de barros los mejor valorados organolépticamente.

Las holandas mejor valoradas sensorialmente fueron las que procedían de suelos de albarizas y con las que se obtuvo mayor volumen de la fracción corazón, sin embargo la producción de uva fue menor que en suelos de arenas, con la misma poda y edad.

Comparando los resultados de los trabajos realizados en las cosechas de 2016 y 2017 se puede decir que, en ambas cosechas, los vinos peor valorados fueron los procedentes de uva cultivada en suelo de arenas. Asimismo, las holandas mejor valoradas fueron las procedentes de uva cultivada en suelo de albarizas.

En cualquier caso, son necesarios más años de estudio para poder obtener resultados más sólidos.

\section{Agradecimientos}

Los resultados presentados han sido obtenidos en el marco del proyecto BESTBRANDY, cuyo título completo es "Factores que influyen en la calidad del Brandy y nuevos sistemas de elaboración del mismo, desde el viñedo al envasado", que cuenta con el apoyo financiero del Centro para el Desarrollo Tecnológico Industrial (CDTI) a través de la convocatoria Interconecta, cofinanciada con fondos FEDER de la Unión Europea.

\section{Bibliografía}

1. M.S. Jurado, Potencial agronómico y enológico de diez variedades blancas de vid como materia prima en la obtención de aguardientes para Brandy de Jerez. Estudio comparativo de las holandas producidas. Tesis Doctoral, Universidad Cádiz (2016)

2. A. García de Luján. La Viticultura de Jerez. ISBN $97-$ 8847-1146-786, (1997)

3. O.I.V. Recueil des méthodes internationales d'analyse des vins et des moûts. Paris. Office International de la Vigne et du Vin (2015)

4. O.I.V. Recueil des méthodes internationales d'analyse des boissons spiritueuses d'origine vitivinicole. Paris. Office International de la Vigne et du Vin (2014)

5. B. Puertas, Estudio del potencial enológico de 15 variedades blancas de vid en la zona del Jerez. Tesis Doctoral, Universidad de Cádiz ISBN 84-7498-3991(1989)

6. J. Muñoz-Ramos, Enciclopedia de los alcoholes. Características y elaboración de licores y aguardientes de todo el mundo. ISBN 84-08-01842-6 (1996)

7. S.C. Diéguez, M.L.G. de la Peña, E.F. Gómez. Volatile composition and sensory characters of commercial Galicia orujo spirits. J. Agric. Food Chem. 53, 6796765 (2005) 